10. Sheiban I, Infantino V, Bollati M. Buddy balloon to deliver a percutaneous aortic valve device: a percutaneous shoehorn. Catheter Cardiovasc Interv. 2009;74:805-7.

Ricardo Allende, Rishi Puri, Éric Dumont, María del Trigo, Omar Abdul-Jawad Altisent, Josep Rodés-Cabau*

Department of Cardiology, Quebec Heart and Lung

Institute, Laval University, Quebec City, Quebec, Canada
* Corresponding author at: Quebec Heart \& Lung Institute, Laval University, 2725 Chemin Sainte-Foy, G1V 4G5 Quebec, QC, Canada.

E-mail address: josep.rodes@criucpq.ulaval.ca

(J. Rodés-Cabau).

http://dx.doi.org/10.1016/j.acmx.2016.01.004

\section{Tetralogía de Fallot con atresia pulmonar y circulación pulmonar poco habitual}

\section{Tetralogy of Fallot with pulmonary atresia and a very unusual pulmonary circulation}

\section{Presentación del caso}

La tetralogía de Fallot con atresia pulmonar es una malformación cardiaca caracterizada por la ausencia de conexión entre el ventrículo derecho y el tronco de la arteria pulmonar, la cual puede ser secundaria a una obstrucción infundibular o valvular; existe también una comunicación interventricular posterior y la aorta puede ser biventricular o nacer totalmente del ventrículo derecho ${ }^{1}$. Lo que hace más interesante esta cardiopatía es lo diversa que puede ser la circulación pulmonar. Se han descrito distintas formas de irrigación de los segmentos pulmonares en esta malformación, pero nunca con el nacimiento de una rama pulmonar desde la arteria coronaria, motivo por el cual presentamos este caso.

Paciente femenina de 6 meses de edad con historia de cianosis generalizada desde el nacimiento; quien ingresó por aumento de la cianosis. Los hallazgos relevantes a la exploración física fueron saturación $76 \%$, soplo continuo infraclavicular izquierdo, pulsos amplios en las 4 extremidades y ligera deformación de lechos ungueales. El electrocardiograma ritmo sinusal, dextrorrotación, crecimiento de cavidades derechas (fig. 1a). La radiografía de tórax con situs solitus bronquial, índice cardiotorácico de 0,61, punta levantada, cono de la pulmonar abombado, rama pulmonar derecha prominente, sin observar la izquierda, arco aórtico izquierdo y oligohemia en ápices pulmonares (fig. 1b). El ecocardiograma mostró tetralogía de Fallot con atresia pulmonar sin visualizar las ramas pulmonares, por lo que se realizó cateterismo cardiaco encontrando presiones ventriculares igualadas $(80 / 10 / 12 \mathrm{mmHg})$, circulación pulmonar dada por ramas pulmonares no confluentes; la derecha naciendo del tronco de la coronaria izquierda (fig. 1c) y la izquierda del conducto arterioso, con estenosis en su origen (fig. 1d). Tenía 2 colaterales aortopulmonares derechas hipertensas; una irrigaba la porción apical del pulmón derecho $(50 / 25 / 33 \mathrm{mmHg})$ y la otra, la porción media y basal ipsilateral $(45 / 25 / 31 \mathrm{mmHg})$. Para caracterizar mejor el origen de la rama pulmonar derecha del tronco de la coronaria izquierda se realizó tomografía que corroboró el diagnóstico (fig. 1e).
Se manejó en 2 tiempos. Primero, embolización de colaterales aortopulmonares con dispositivos coils, y 6 días después se realizó cirugía de Rastelli, sección y sutura del origen de ambas ramas pulmonares confluyéndolas a la porción distal del injerto de yugular bovino (Contegra ${ }^{\circledR}$ de $14 \mathrm{~mm}$ ), plastia en origen de rama pulmonar izquierda y cierre de la comunicación interventricular. Después de una mala evolución en el posquirúrgico se decide llevar nuevamente a cateterismo, encontrando estenosis en origen de rama pulmonar izquierda con gradiente de $35 \mathrm{mmHg}$ y relación de presiones entre ventrículo derecho e izquierdo $(\mathrm{pVD} / \mathrm{pVl})$ de 0,62. Se realizó angioplastia con stent de rama izquierda (fig. 2a), con registro de presiones final (VD $35 / 6 / 12 \mathrm{mmHg}$ vs VI $80 / 4 / 11 \mathrm{mmHg}$ ). Evolucionó hacia la mejoría con egreso 4 días después. La tomografía control sin zonas de estenosis en tronco ni ramas pulmonares (fig. $2 \mathrm{~b}$ y 2c).

Actualmente, la paciente se encuentra en clase funcional I, bajo seguimiento a través de consulta externa.

La tetralogía de Fallot con atresia pulmonar es una entidad que requiere conocimiento del desarrollo embrionario. Las porciones proximales de las ramas de la arteria pulmonar habitualmente se originan a partir de los sextos arcos aórticos y las colaterales aortopulmonares desde la aorta descendente ${ }^{1}$. El nacimiento aórtico de la rama derecha se origina por una malposición del tabique aortopulmonar de Tandler que separa el cuarto arco aórtico izquierdo (origen del cayado aórtico) de los sextos arcos aórticos. Normalmente este tabique tiene una orientación inclinada de derecha a izquierda en sentido dorsoventral ${ }^{2}$. Proponemos como posible origen embriológico del nacimiento aórtico de una rama pulmonar, a la malposición del tabique aortopulmonar, el cual sería ectópico en sentido dorsoventral que determinaría que la rama pulmonar derecha naciera de la aorta ascendente y la rama izquierda del tronco pulmonar. La rama derecha puede cambiar de posición de manera descendente hasta llegar a la arteria coronaria izquierda tal como se ha descrito en este caso (fig. 2d).

En esta malformación la clínica depende de la circulación pulmonar. Si esta es unifocal (por un conducto arterioso) la cianosis marcada e hipoxemia aparecerán a edades tempranas pudiendo ocurrir la muerte si hay cierre del mismo ${ }^{3}$. Cuando es multifocal (colaterales aortopulmonares) las manifestaciones clínicas serán tardías pudiendo llegar a la vida adulta. Debido a esta diversidad de circulación pulmonar el cateterismo es obligatorio, ya que además de mostrar la anatomía informará presiones de las colaterales ${ }^{4}$. 

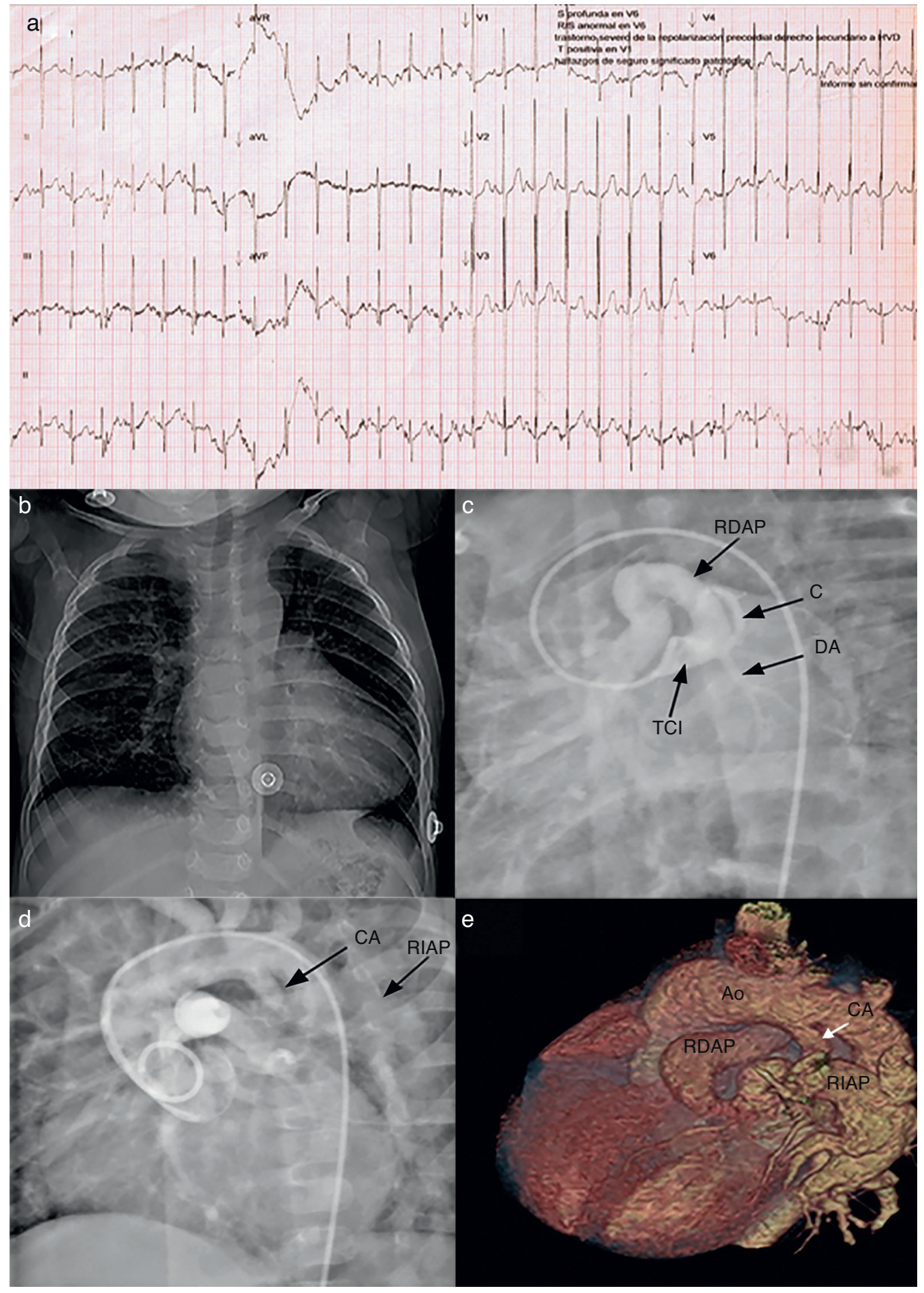

Figura 1 1a) Electrocardiograma que muestra crecimiento de cavidades derechas; 1b) Radiografía de tórax que muestra RDAP dilatada; 1c) Angiografía que revela el nacimiento de la RDAP del TCl; 1d) Nacimiento de la RIAP desde CA, y 1e) Reconstrucción tridimensional de tomografía de tórax que evidencia el nacimiento de las ramas pulmonares.

Decidir el tipo de cirugía (paliativa o definitiva) a realizar en esta cardiopatía es difícil, y la elección dependerá de factores anatómicos, básicamente relacionados con la estructura de las arterias pulmonares. En nuestro caso se optó por la corrección total debido a la existencia de ramas pulmonares centrales, con área central $>50 \%$ del valor normal para la edad y superficie corporal; además dichas ramas irrigaban ambos pulmones en su totalidad ${ }^{5}$.

Por otro lado, cuando en la etapa posquirúrgica la evolución es desfavorable, se debe sospechar de la presencia de cortocircuitos residuales o gradiente obstructivo entre el ventrículo derecho y las arterias pulmonares distales. En 

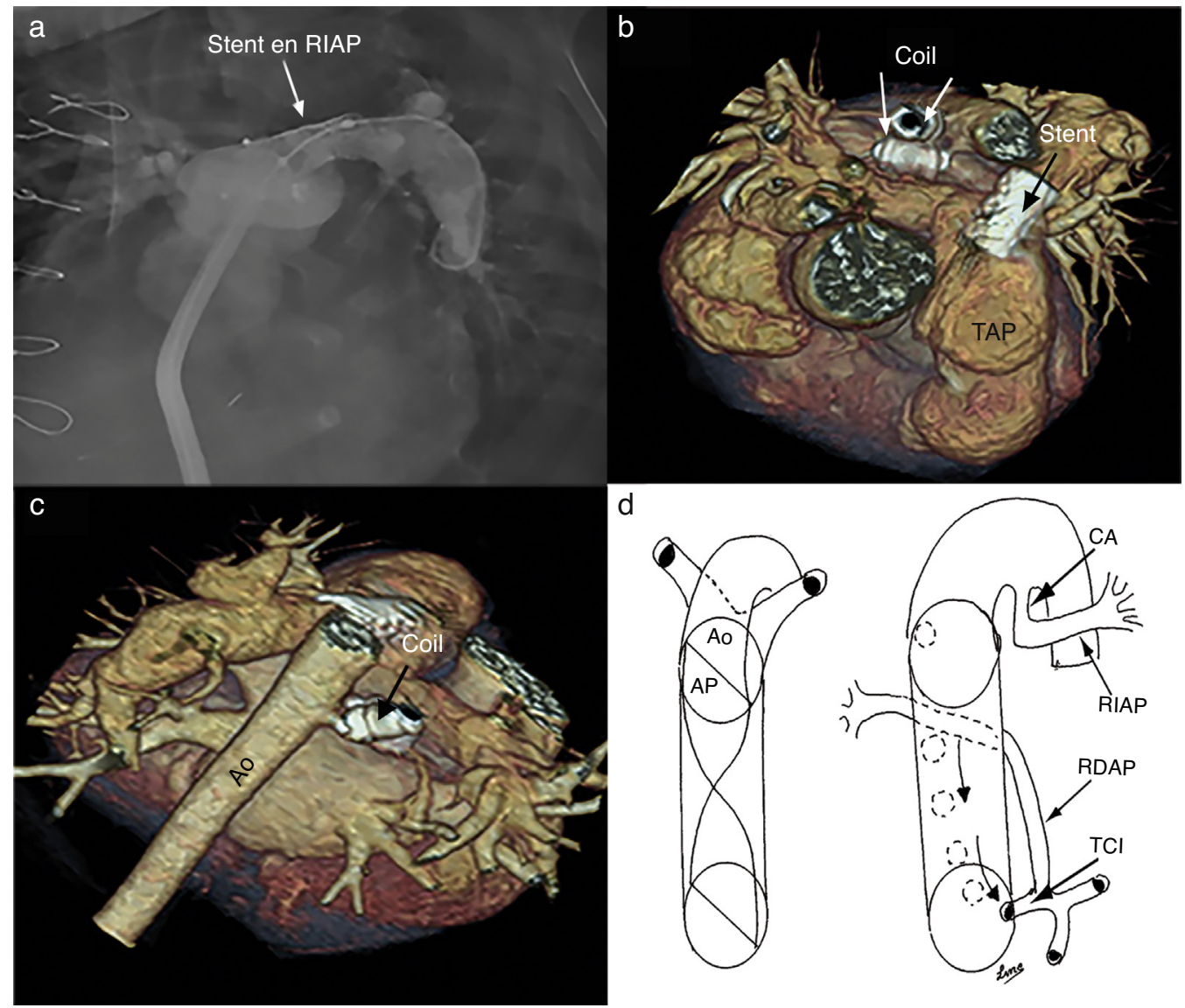

Figura 2 2a) Angiografía que muestra la colocación de stent en origen de RIAP; 2b y 2c) Reconstrucción tridimensional de tomografía de tórax que muestra stent en RIAP y coils en colaterales aortopulmonares derechas, y 2d) Esquema que muestra el desarrollo embriológico del nacimiento de la RDAP desde el TCl.

nuestro caso, el deterioro clínico fue secundario a estenosis en el origen de la rama pulmonar izquierda, la cual se pudo resolver mediante cateterismo obteniendo así una relación $\mathrm{pVD} / \mathrm{pVI}<0,5$ considerando el resultado como adecuado ${ }^{5}$.

El excelente resultado quirúrgico en esta cardiopatía depende de una buena caracterización de la irrigación pulmonar. Propusimos una posible teoría embriológica como causal de esta entidad, antes no descrita, así como una técnica quirúrgica que llevó a la corrección exitosa. Por otro lado, es necesario el trabajo multidisciplinario en el tratamiento de estos pacientes, ya que con el avance a pasos agigantados del cateterismo intervencionista se pueden resolver complicaciones posquirúrgicas disminuyendo la morbimortalidad que implicaría una segunda cirugía.

\section{Bibliografía}

1. Muñoz Castellanos L, Ramírez Marroquín S, Kuri Nivon M. Tetralogía de Fallot con atresia pulmonar: morfopatología y anatomía quirúrgica. Arch Cardiol Méx. 2010;80:141-53.

2. Curi-Curi P, Ramírez S, Muñoz L, et al. Origen anómalo de la rama pulmonar izquierda a partir de la aorta ascendente en un niño con estenosis subaórtica asociada. Arch Cardiol Méx. 2010;80:187-91.

3. Franco J, Sandoval N, Carrillo G, et al. Atresia pulmonar con comunicación interventricular. En: Díaz G, Sandoval N, Vélez

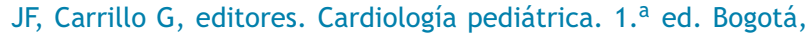
Colombia: McGraw Hill; 2003. p. 551-64.

4. Griffiths $S$, Levine 0 , Andersen D. Aortic origin of the right pulmonary artery. Circulation. 1962;25:73-84.

5. Calderón-Colmenero J, Buendía-Hernández A. En: Attie F, Calderón J, Zabal C, et al. (editores). Cardiología pediátrica. 2. ${ }^{a}$ ed. México, D.F. Médica Panamericana; 2013. p. 223-31.

Aldo Campos-Quintero ${ }^{a, *}$, Irma Miranda-Chávez ${ }^{a}$, Roberto Mijangos-Vázquez ${ }^{a}$, Luis Muñoz-Castellanos ${ }^{\mathrm{b}}$, Samuel Ramírez-Marroquín ${ }^{c}$ y Alfonso Buendía-Hernández ${ }^{a}$

\section{a Departamento de Cardiología Pediátrica, Instituto Nacional de Cardiología Ignacio Chávez, México, D.F., México \\ b Departamento de Cirugía de Malformaciones Congénitas del Corazón, Instituto Nacional de Cardiología Ignacio Chávez, México, D.F., México \\ ' Departamento de Embriología, Instituto Nacional de Cardiología Ignacio Chávez, México, D.F., México}

* Autor para correspondencia. Juan Badiano, 1, Col. Sección XVI, Del. Tlalpan, México, D.F., México.

Teléfono: +52 557329 11, ext.: 1336 .

Correo electrónico: aldocampos06@gmail.com

(A. Campos-Quintero).

http://dx.doi.org/10.1016/j.acmx.2015.07.005 